\title{
Brain-Derived Neurotrophic Factor Regulation of Retinal Growth Cone Filopodial Dynamics Is Mediated through Actin Depolymerizing Factor/Cofilin
}

\author{
Scott Gehler, ${ }^{1}$ Alisa E. Shaw, ${ }^{2}$ Patrick D. Sarmiere, ${ }^{2}$ James R. Bamburg, ${ }^{2}$ and Paul C. Letourneau ${ }^{1}$ \\ ${ }^{1}$ Department of Neuroscience, University of Minnesota, Minneapolis, Minnesota 55455, and 2Department of Biochemistry and Molecular Biology, Colorado \\ State University, Fort Collins, Colorado 80523
}

\begin{abstract}
The molecular mechanisms by which neurotrophins regulate growth cone motility are not well understood. This study investigated the signaling involved in transducing BDNF-induced increases of filopodial dynamics. Our results indicate that BDNF regulates filopodial length and number through a Rho kinase-dependent mechanism. Additionally, actin depolymerizing factor (ADF)/cofilin activity is necessary and sufficient to transduce the effects of BDNF. Our data indicate that activation of ADF/cofilin mimics the effects of BDNF on filopodial dynamics, whereas ADF/cofilin inactivity blocks the effects of BDNF. Furthermore, BDNF promotes the activation of ADF/ cofilin by reducing the phosphorylation of ADF/cofilin. Although inhibition of myosin II also enhances filopodial length, our results indicate that BDNF signaling is independent of myosin II activity and that the two pathways result in additive effects on filopodial length. Thus, filopodial extension is regulated by at least two independent mechanisms. The BDNF-dependent pathway works via regulation of $\mathrm{ADF} /$ cofilin, independently of myosin II activity.
\end{abstract}

Key words: filopodia; growth cone; neurotrophin; BDNF; ADF/cofilin; myosin

\section{Introduction}

Neuronal growth cones encounter extrinsic cues and make pathway choices that are vital to properly wiring synaptic connections. These choices are mediated by dynamic sensory structures, lamellipodia and filopodia. Filopodia are particularly important in detecting guidance cues (Gomez and Letourneau, 1994; Kuhn et al., 1994; Dickson, 2002). Signaling by guidance cues can begin within filopodia (Davenport et al., 1993; Robles et al., 2003), and a single filopodial contact is sufficient to turn a growth cone (O'Connor et al., 1990). Growth cones that lack filopodia exhibit impaired guidance (Bentley and Toroian-Raymond, 1986; Chien et al., 1993; Zheng et al., 1996). In addition, dendritic and axonal filopodia participate in morphogenesis of terminals and synaptogenesis (Jontes and Smith, 2000; Niell et al., 2004). Thus, filopodial dynamics are important to axonal guidance and synaptogenesis.

Among their diverse activities, neurotrophins regulate growth cone motility and guidance (Gundersen and Barrett, 1979; Gallo et al., 1997; Alsina et al., 2001; Song and Poo, 2001; Tucker et al., 2001; McQuillen et al., 2002; Gehler et al., 2004; Glebova and Ginty, 2004). Neurotrophins bind two receptor types, the selec-

Received July 14, 2004; revised Sept. 2, 2004; accepted 0ct. 14, 2004.

This work was supported by grants from the National Institutes of Health (NIH) (HD19950 to P.C.L.; GM35126 and NS40371 to J.R.B.), the National Science Foundation (IBN0080932), and the Minnesota Medical Foundation. S.G. is a trainee on an NIH Eye Institute Training Grant. P.D.S. was supported by the Christopher Reeve Paralysis Foundation.

Correspondence should be addressed to Dr. Paul C. Letourneau, Department of Neuroscience, University of Minnesota, 6-145 Jackson Hall, Minneapolis, MN 55455. E-mail: letour@med.umn.edu.

DOI:10.1523/JNEUROSCI.2836-04.2004

Copyright $\odot 2004$ Society for Neuroscience ～0270-6474/04/2410741-09\$15.00/0 tive Trk receptor tyrosine kinases and the p75 pan-neurotrophin receptor. Induction of growth cone turning by neurotrophins involves a Trk-dependent mechanism (Gallo et al., 1997; Song and Poo, 2001), whereas neurotrophins regulate filopodial dynamics through a p75-dependent mechanism (Gehler et al., 2004).

Filopodial dynamics involve complex regulation of actin filaments. Filopodial and lamellipodial protrusion involves a balance between actin polymerization, retrograde filament flow, and actin depolymerization (Mallavarapu and Mitchison, 1999). These activities are targets of Rho GTPases (Dickson, 2001). Rho kinase (ROCK) is an effector of RhoA that regulates actin dynamics in several ways. ROCK activates LIM kinase (LIMK), which phosphorylates actin depolymerizing factor (ADF)/cofilin, inhibiting its activity (Agnew et al., 1995; Arber et al., 1998; Yang et al., 1998; Maekawa et al., 1999; Meng et al., 2002). ADF and cofilin belong to a conserved family of actin-associated proteins that enhance actin dynamics (Bamburg, 1999). ADF/cofilin binds to actin filaments (F-actin) stabilizing a twisted form of the filament (McGough et al., 1997; Galkin et al., 2001). Consequently, the off rate of actin monomers (Carlier et al., 1997) and filament severing are increased (McGough et al., 1997; Maciver, 1998; Bobkov et al., 2004). Overexpression of ADF/cofilin enhances neurite outgrowth, actin dynamics, and growth cone dynamics (Meberg and Bamburg, 2000; Endo et al., 2003). Furthermore, growth cone collapse induced by semaphorin $3 \mathrm{~A}$ is dependent on transient phosphorylation of cofilin by LIMK (Aizawa et al., 2001).

Besides signaling through LIMK, ROCK may also regulate 
growth cone motility by regulating myosin II activity (Amano et al., 1996; Kimura et al., 1996). Acute inactivation of myosin IIB leads to reduced growth cone area and loss of lamellipodia (Diefenbach et al., 2002). A reduced growth cone area was also observed for neurons of myosin IIB knock-out mice, but no changes in filopodial extension or retraction were reported (Bridgman et al., 2001; Brown and Bridgman, 2003). Thus, although signaling downstream of ROCK can regulate both myosin II and ADF/ cofilin, it is unclear how ROCK is involved in regulating filopodial motility.

Previously, we showed that BDNF regulates filopodial length through a RhoA-dependent pathway (Gehler et al., 2004). To further characterize how neurotrophins regulate filopodial dynamics, we investigated signaling downstream from RhoA. We show that the effects of BDNF are mimicked by inhibiting ROCK. In addition, our evidence implicates $\mathrm{ADF} /$ cofilin in mediating the effects of BDNF. Although inhibition of myosin II also enhances filopodial length, BDNF does not signal through myosin II to regulate filopodial dynamics.

\section{Materials and Methods}

Reagents. BDNF was generously provided by Regeneron Pharmaceuticals (Tarrytown, NY). Laminin was purchased from Invitrogen (Carlsbad, CA). The ROCK inhibitors Y-27632, HA1077, and H-1152 were all obtained from Calbiochem (San Diego, CA). Blebbistatin was obtained from Toronto Research Chemicals (North York, Ontario, Canada). All culture media were obtained from Life Technologies (Rockville, MD), and other reagents were obtained from Sigma (St. Louis, MO), unless specified. The chick p75 antibody (CHEX) was a generous gift from Dr. Louis Reichardt (University of California at San Francisco, San Francisco, CA).

Mutagenesis, production, and purification of recombinant proteins. The XAC1 cDNA in a pGEX vector (Abe et al., 1996) was digested with NcoI and HindIII and inserted into a pETMWT7 expression plasmid. The wild-type XAC1 was mutagenized to change two lys codons (AAG AAA) for residues 95 and 96 to glutamine codons (CAG CAA) using the Quik Change Mutagenesis kit (Stratagene, La Jolla, CA). The forward primer was 5'-GCACTCTACGAGACAAAGGAAACACAGCAAGAGGATCTTG-3', and the reverse primer was $5^{\prime}$-CAAGATCCTCTTGCTGTGTTTCCTTTGTCTCGTAGAGTGC-3'. The resultant clones were double-strand sequenced throughout the entire XAC1 coding region and showed only the desired changes. pET vectors for expressing XAC(KK95,96QQ), XAC(3A), and XAC(3E) (Meberg and Bamburg, 2000) as well as glutathione $S$ -

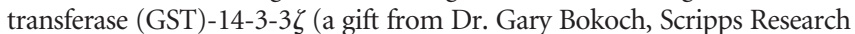
Institute, La Jolla, CA) were also placed into Escherichia coli BL21DE3, and in a 11 culture of bacteria $\left(0.95 \mathrm{OD}_{600 \mathrm{~nm}}\right)$, IPTG (isopropyl $\beta$-D-1-thiogalactopyranoside; $0.5 \mu \mathrm{M}$ ) was used to induce protein expression. After $3 \mathrm{hr}$, bacteria were pelleted and stored frozen until used.

All bacterial pellets were resuspended in $20 \mathrm{ml}$ of Tris-EDTA, $\mathrm{pH} 7.5$, at $25^{\circ} \mathrm{C}$ and incubated with lysozyme and protease inhibitors to lyse the cells. DNA was digested with DnaseI, and the supernatant was clarified by centrifugation. For purification of GST-14-3-3 $\zeta$, the extract was passed over a $2 \mathrm{ml}$ column of glutathione-Sepharose, washed with buffer, and GST-14-3-3 $\zeta$ was eluted with glutathione according to the manufacturer's procedure. Samples were dialyzed against $10 \mathrm{~mm}$ Tris and $0.5 \mathrm{~mm}$ DTT before storage by quick freezing in liquid nitrogen. For purification of the $\mathrm{XAC}(3 \mathrm{~A})$ and $\mathrm{XAC}(3 \mathrm{E})$ mutant proteins, the clarified bacterial extract was passed through $6 \mathrm{ml}$ of DEAE-cellulose in $10 \mathrm{~mm}$ Tris and 5 mM DTT, $\mathrm{pH}$ 7.5. The flow-through was then passed over a $6 \mathrm{ml}$ column of Green A-agarose resin (Millipore, Bedford, MA) in the same buffer. After washing with 10 column volumes, the purified XAC proteins were eluted with a gradient of $0-200 \mathrm{~mm} \mathrm{NaCl}$ in the same Tris buffer. The protein peak was identified, concentrated with UltraFree- 15 concentrators (Millipore), dialyzed against $10 \mathrm{~mm}$ Tris, $\mathrm{pH} 7.5$, and $5 \mathrm{~mm}$ DTT, and quick-frozen. For purification of the XAC(KK95,96QQ) mutant, the above procedure was followed, except the mutant protein did not bind to the Green A resin. The flow-through from this column was adjusted to pH 6.8 using 1 м PIPES buffer at pH 6.8 (final PIPES concentration, 25 $\mathrm{mm}$ ). This sample was passed over a $6 \mathrm{ml}$ column of Green A resin equilibrated in $20 \mathrm{~mm}$ PIPES, $10 \mathrm{~mm}$ Tris, and $5 \mathrm{~mm}$ DTT, pH 6.8. After washing with the same buffer, the protein was eluted with a $0-200 \mathrm{~mm}$ $\mathrm{NaCl}$ gradient. Fractions containing the KKQQ mutant were identified by SDS-PAGE, pooled, and the buffer was exchanged to $10 \mathrm{~mm}$ Tris and 5 mм DTT by dilution and reconcentration, before quick-freezing. Homogeneity of all proteins estimated by SDS-PAGE and Coomassie blue staining was $97-99 \%$.

Tissue culture. Retina was dissected from embryonic day 7 white Leghorn chicken embryos. Explants were cultured on glass coverslips coated with $25 \mu \mathrm{g} / \mathrm{ml}$ laminin. Retinal explants were cultured in F12 medium supplemented with additives, as described previously (Ernst et al., 2000).

Protein loading. Proteins were delivered into cells using Chariot reagent (Active Motif, Carlsbad, CA). Protein delivery was accomplished according to the manufacturer's suggestions, as described previously (Gallo et al., 2002a,b). Briefly, Chariot was complexed with $1 \mu \mathrm{g}$ of $\mathrm{XAC}(3 \mathrm{~A}), \mathrm{XAC}(3 \mathrm{E}), \mathrm{XAC}(\mathrm{KK} 95,96 \mathrm{QQ}), \mathrm{GST}-14-3-3 \zeta$, or bovine serum albumin. After protein loading, cultures were subsequently exposed to experimental conditions or fixed with $0.25 \%$ glutaraldehyde.

Phospho-ADF/cofilin staining. Cultures were fixed with $4 \%$ paraformaldehyde and $10 \%$ sucrose for $1 \mathrm{hr}$. Cells were quenched with $0.1 \mathrm{M}$ glycine, then extracted with $0.5 \%$ Triton X-100 for $10 \mathrm{~min}$. After rinse, cultures were blocked with $10 \%$ normal goat serum for $1 \mathrm{hr}$ at room temperature, followed by incubation with 1:200 rabbit anti-phosphoADF/cofilin (pAC) (Meberg et al., 1998) for $1 \mathrm{hr}$. After rinse, cultures were incubated with 1:800 Alexa488 goat anti-rabbit secondary antibody plus 1:20 Alexa568-conjugated phalloidin (Molecular Probes, Eugene, OR) in block for $30 \mathrm{~min}$ and then mounted with No Fade. Growth cones were quantified for pAC intensity by tracing individual growth cones and taking the total intensity minus background using MetaVue (Universal Imaging, West Chester, PA).

Time-lapse video microscopy. Phase-contrast images were collected using an Olympus XC-70 inverted microscope with a $60 \times$ objective equipped with a SPOT digital camera (Diagnostic Instruments, Sterling Heights, MI). Images were acquired every $20 \mathrm{sec}$ during a $20 \mathrm{~min}$ recording session. Digital images were collected using MetaVue imaging software (Universal Imaging). Filopodial dynamics were quantified by measuring extension or retraction of individual filopodial tips during sequential frames, as described by Gehler et al. (2004).

A motility index for filopodial dynamics was used to determine the amount of time a filopodium spent extending or retracting, as opposed to remaining stationary. The distal $20 \mu \mathrm{m}$ of the growth cone was analyzed, and filopodia were assayed in sequential image intervals. Criteria for this assay included the following: (1) a filopodium was scored as moving if it had extended or retracted in sequential frames at 20 sec intervals; (2) a filopodium that did not exhibit any displacement from the previous frame was scored as stationary; (3) only existing filopodia were scored; a newly formed filopodium was not scored during the frame in which it appeared but was scored in subsequent frames; (4) a filopodium that detached from the substrate was scored as moving only if the filopodium was attached in the previous frame; and (5) a filopodium was scored as stationary if an associated lamellipodium moved but there was no change in the displacement of the filopodial tip. The motility index was plotted in 2 min time bins as a percentage of the number of moving filopodia relative to total filopodia during each $20 \mathrm{sec}$ time interval.

Image acquisition and analysis. Fluorescent images were collected using an Olympus XC-70 inverted microscope. Growth cones were visualized with a $60 \times$ objective, and images were obtained using a SPOT digital camera. Image analysis was accomplished using MetaVue imaging software (Universal Imaging). Growth cones were enhanced to $150 \%$ magnification, and individual filopodia were measured using the digital software. Only filopodia within $20 \mu \mathrm{m}$ of the distal tip of the growth cone were counted and measured. Filopodial length and number were determined per growth cone. 

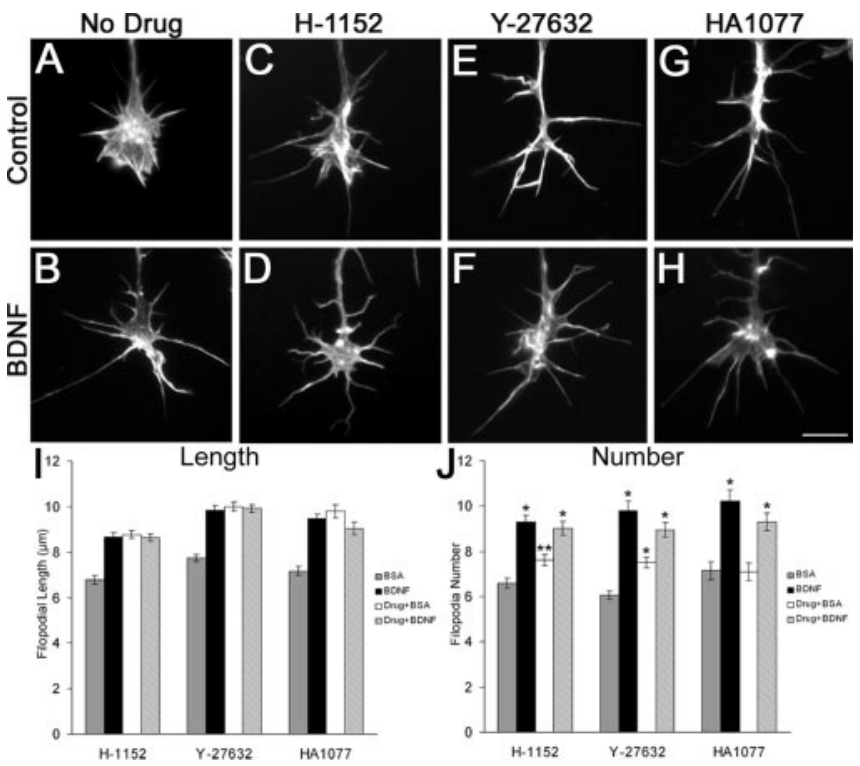

Figure 1. BDNF regulates filopodial dynamics through a ROCK-dependent pathway. $A-H$, Phalloidin staining of F-actin in example growth cones after treatment with three ROCK inhibitors. Inhibition of ROCK with $500 \mathrm{~nm} \mathrm{H}-1152$ or $50 \mu \mathrm{m} \mathrm{HA} 1077$ for 30 min, or $10 \mu \mathrm{M}$ Y-27632 for $1 \mathrm{hr}(C, E, G)$, enhanced filopodial length by $29-37 \%$ relative to control $(A)$. The addition of 160 пм BDNF for 30 min to drug-treated cultures had no additive effect on filopodial length $(B, D, F$, $H, I)$. Scale bar, $10 \mu \mathrm{m}$. J, ROCK inhibitors have a minor effect on filopodial number, but BDNF treatment of drug-treated cultures further enhanced the number to levels similar to BDNF treatment alone. Data are mean \pm SEM from a minimum of three independent experiments. ${ }^{*} p<0.001 ;{ }^{* *} p<0.005$; statistical difference relative to control. For all treatments in $I, p<$ 0.001 indicates statistical significance relative to control (two-sample $t$ test).

\section{Results}

BDNF regulates filopodial dynamics through ROCK

We have previously shown that neurotrophins regulate filopodial dynamics through a p75-dependent pathway in which BDNF reduced RhoA activity within growth cones (Gehler et al., 2004). To test the idea that the consequent reduced signaling by the RhoA effector ROCK is involved in BDNF regulation of filopodial dynamics, we treated chick retinal cultures with three ROCK inhibitors: $500 \mathrm{~nm} \mathrm{H}-1152$ or $50 \mu \mathrm{M} \mathrm{HA1077}$ for $30 \mathrm{~min}$, or $10 \mu \mathrm{M}$ Y-27632 for $1 \mathrm{hr}$. All three inhibitors enhanced filopodial length by $29-37 \%$ over controls (Fig. $1 A-I$ ). In addition, $\mathrm{H}-1152$ and Y-27632 produced a 33\% and 19\% enhancement, respectively, of filopodial length on dorsal root ganglion (DRG) cell growth cones (our unpublished results). The increased filopodial lengths resulting from ROCK inhibition were not a consequence of the retraction of lamellipodia. Growth cones treated with BDNF or ROCK inhibitors exhibited intact lamellipodia, whereas we previously showed that the increased filopodial lengths induced by BDNF occurred through increases in the extension of filopodial tips, not by the withdrawal of the leading edge at the bases of filopodia (Gehler et al., 2004) (Fig. 5J). The addition of $160 \mathrm{~nm}$ BDNF for 30 min to these drug-treated cultures did not further increase filopodial length, suggesting that BDNF signals through reducing ROCK activity to regulate filopodial length.

Filopodial number was also increased by the inhibition of ROCK. Treatment of retinal neurons with H-1152 or Y-27632 produced a modest increase in the number of filopodia, whereas HA1077 had no effect (Fig. 1J). The addition of BDNF to drugtreated cultures produced additional increases of filopodia number to levels that were similar to BDNF treatment alone. These results suggest filopodial number is at least partially regulated
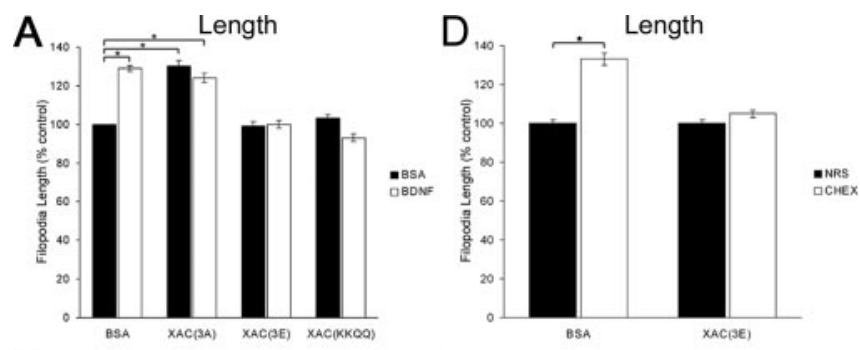

B

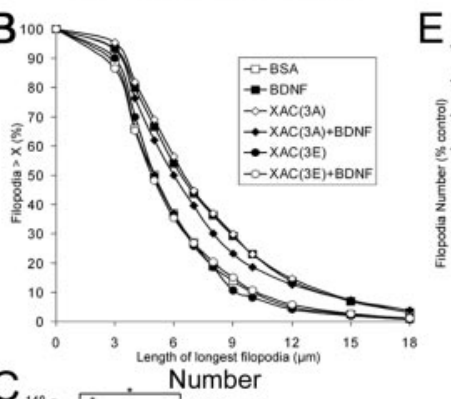

E Number

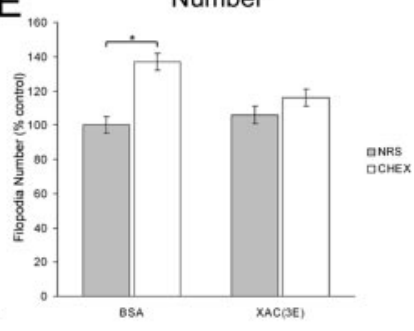

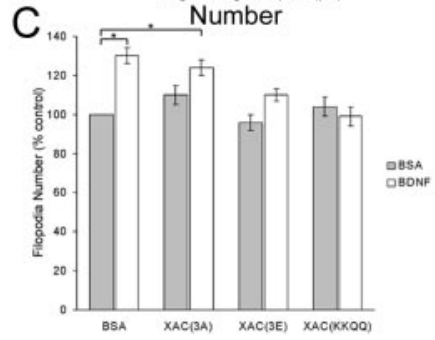

Figure 2. ADF/cofilin is necessary and sufficient to transduce the effects of BDNF on filopodial dynamics. $A, X A C(3 A)$ was sufficient to enhance filopodial length to levels that mimicked BDNF treatment. BDNF had no additive effect on length on XAC(3A)-loaded growth cones. $X A C(3 E)$ and $X A C(K K 95,960 Q)$ alone had no effect on filopodial length but both blocked the stimulatory effects of BDNF. B, BDNF, XAC( $(3 A)$, and $X A C(3 A)$ plus BDNF increased all filopodia lengths as indicated by a rightward shift of the length distribution curve. $C$, Although $X A C(3 A)$ alone did not enhance filopodial number to levels similar as BDNF treatment alone, $\mathrm{XAC}(3 \mathrm{E})$ and $\mathrm{XAC}(\mathrm{KKQQ})$ blocked the BDNF-induced increases on filopodial number. D, E, BDNF-induced regulation of ADF/cofilin is mediated through p $75{ }^{\text {NTR }}$. CHEX-induced increases on filopodial length and number were blocked by XAC(3E). Data were presented as percentage of control \pm SEM from a minimum of four independent experiments. ${ }^{*} p<0.001$; statistical difference relative to control (two-sample $t$ test).

through a ROCK-independent mechanism, but additional BDNF signaling may be involved in regulating filopodial number.

$\mathrm{ADF} /$ cofilin activity is required to transduce BDNF-mediated effects on filopodial dynamics

ROCK signaling activates LIMK, which phosphorylates ADF/cofilin. To study the role of ADF/cofilin in regulating filopodial dynamics, we used a peptide-based transfection system that allowed the introduction of $\mathrm{ADF} / \mathrm{cofilin}$ mutant proteins into neuronal growth cones. Introduction of a constitutively active form of $\mathrm{ADF} / \mathrm{cofilin}[\mathrm{XAC}(3 \mathrm{~A})]$ into chick retinal growth cones caused a $30 \%$ increase on filopodial length (Fig. $2 \mathrm{~A}$ ). Conversely, introduction of a pseudophosphorylated (less active) form [XAC(3E)] had no effect on growth cone morphology. Next, we determined whether BDNF treatment affected filopodial dynamics of XACtreated growth cones. BDNF treatment of XAC(3A)-containing growth cones did not further enhance filopodial length, compared with $\mathrm{XAC}(3 \mathrm{~A})$ treatment alone. Conversely, the normally stimulatory effects of BDNF were blocked by the inactive $\mathrm{XAC}(3 \mathrm{E})$, suggesting $\mathrm{BDNF}$ regulates filopodial dynamics by activating $\mathrm{ADF} /$ cofilin. $\mathrm{BDNF}$ and $\mathrm{XAC}(3 \mathrm{~A})$ caused about the same 
percentage increase in all filopodia lengths, as indicated by the rightward shift of the length distribution curve (Fig. $2 B$ ).

$\mathrm{ADF} /$ cofilin promotes actin turnover and reorganization through two mechanisms. One mechanism is through binding along actin filaments and severing them, whereas the other mechanism involves promoting polymer loss from filament pointed ends, thereby increasing the pool of actin monomer. To determine whether the severing activity of $\mathrm{ADF} /$ cofilin was required in transducing the effects of BDNF, we tested the effects of a nonsevering mutant of ADF/cofilin, XAC(KK95,96QQ) (Pope et al., 2000). Incorporation of KKQQ alone had no effect on filopodial length, but KKQQ blocked the BDNF-induced increases on filopodial length (Fig. 2A). These results suggest that the filament-severing activity of $\mathrm{ADF} /$ cofilin is necessary to mediate the effects of BDNF on filopodial length.

Filopodia number was also affected by incorporation of ADF/ cofilin mutants. Although XAC(3A) increased filopodial length to a similar extent as BDNF treatment, $\mathrm{XAC}(3 \mathrm{~A})$ had a lesser effect on filopodia number (Fig. 2C). The addition of BDNF to $\mathrm{XAC}(3 \mathrm{~A})$-treated cultures further increased the mean filopodia number to levels similar as BDNF treatment alone. XAC $(3 \mathrm{E})$ had no effect on filopodial number, but it blocked most of the effects of BDNF. Furthermore, KKQQ blocked the BDNF-induced increases on filopodial number, while having no effect by itself. These results suggest that ADF/cofilin activity is necessary but not sufficient for the BDNF-induced increase in filopodial number.

We have previously shown that BDNF regulates filopodial dynamics through the p75 neurotrophin receptor (Gehler et al., 2004). To determine whether ADF/cofilin activity is regulated through a p75-dependent mechanism, we used an anti-chick p75 antibody (CHEX), which we have previously shown mimics the effects of BDNF on filopodial dynamics. Treatment with CHEX alone caused a $33 \%$ and $37 \%$ increase in filopodial length and number, respectively (Fig. $2 D, E)$. Incorporation of XAC $(3 \mathrm{E})$ blocked CHEX-mediated increases in both length and number. These results provide additional evidence that BDNF-induced regulation of $\mathrm{ADF} /$ cofilin activity is mediated through a p75dependent mechanism.

\section{$\mathrm{BDNF}$ reduces the phosphorylation levels of $\mathrm{ADF} /$ cofilin}

The activity of ADF/cofilin is regulated by the phosphorylation state of serine 3, such that dephosphorylation activates ADF/ cofilin. To test the effect of BDNF on the phosphorylation state of $\mathrm{ADF} /$ cofilin, we conducted quantitative immunofluorescence on retinal growth cones using a phosphospecific antibody to $\mathrm{ADF} /$ cofilin (pAC). Control-treated retinal growth cones displayed robust staining patterns for pAC (Fig. $3 A$ ). Treatment of growth cones with BDNF for 5,10 , or 30 min caused a time-dependent reduction in pAC staining (Fig. $3 A-I$ ). There was a $23 \%$ reduction in pAC signal after treatment with BDNF for $5 \mathrm{~min}$ (Fig. $3 C, D)$, and anti-pAC staining was further reduced by $49 \%$ after a 30 min treatment with BDNF (Fig. $3 G, H$ ). pAC levels are regulated through a ROCK-dependent pathway, presumably via LIMK. Treatment of retinal growth cones with ROCK inhibitors, either $500 \mathrm{~nm} \mathrm{H}-1152$ or $10 \mu \mathrm{M}$ Y-27632, caused a $47 \%$ and $45 \%$ reduction in pAC staining, respectively, compared with control growth cones (Fig. 3J-P). These results suggest that $\mathrm{BDNF}$ regulates filopodial dynamics through downregulation of pAC by way of a ROCK-dependent mechanism.
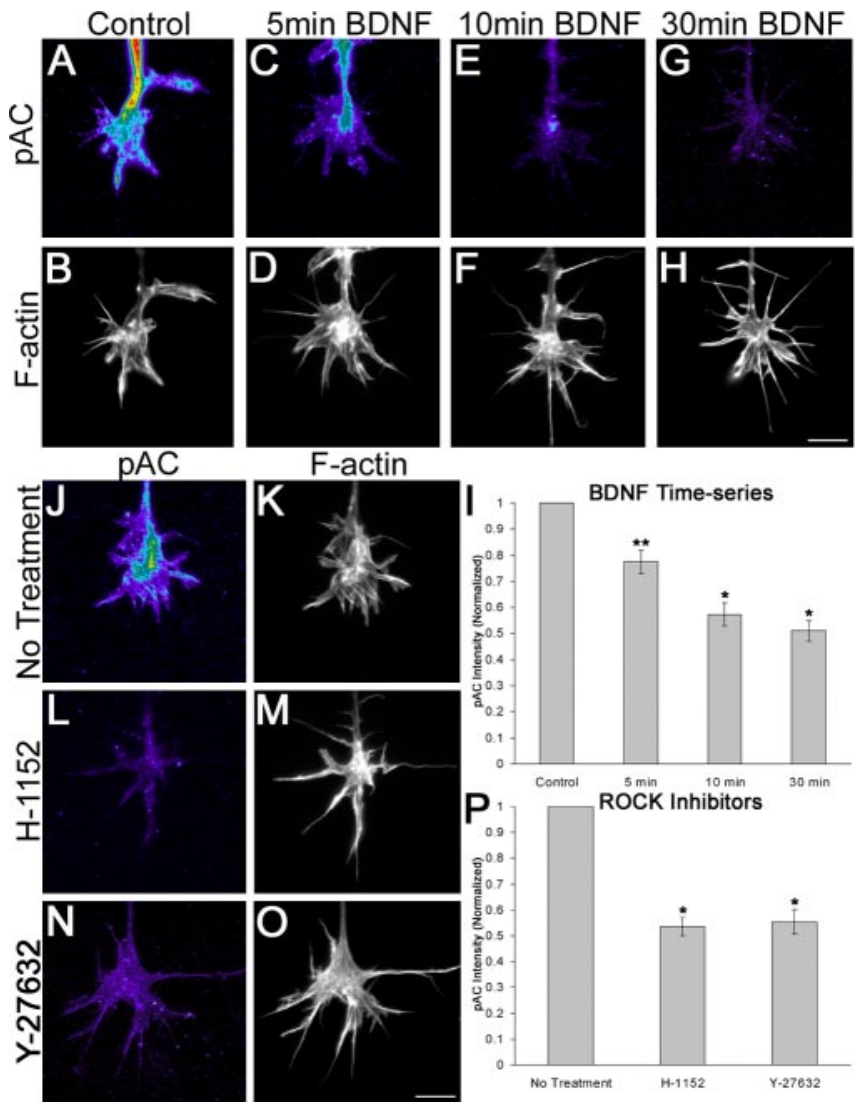

Figure 3. BDNF causes a reduction in $\mathrm{pAC}$ levels on retinal growth cones that is mediated through ROCK. Growth cones treated with BDNF for up to 30 min displayed a gradual reduction in pAC staining levels $(A, C, E, G)$. Growth cones were counterstained using Alexa568-conjugated phalloidin $(B, D, F, H) . I$, Thirty-minute treatment with BDNF caused a $49 \%$ reduction in $\mathrm{PAC}$ levels relative to control treatment. $J-0$, ROCK inhibition with $\mathrm{H}-1152$ or $\mathrm{Y}-27632$ mimicked the effects of BDNF treatment on pAC levels. $P$, Treatment with $\mathrm{H}-1152$ for 30 min or Y-27632 for 1 hr produced a 47 and $45 \%$ reduction, respectively, in pAC levels. Measurements were taken from at least 77 growth cones from a minimum of three independent experiments. Scale bars, $10 \mu \mathrm{m} .{ }^{*} p<0.001 ;{ }^{* *} p<0.005$; statistical significance relative to control (two-sample $t$ test).

\section{The 14-3-3 $\zeta$ protein blocks the effects of BDNF on} filopodial dynamics

The 14-3-3 proteins have regulatory roles in signal transduction by binding to phosphoserine-containing cellular proteins ( $\mathrm{Fu}$ et

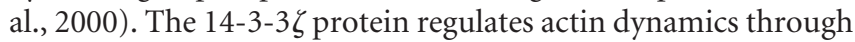
binding and stabilization of inactive phosphocofilin in cell lines (Gohla and Bokoch, 2002). Because BDNF downregulated pAC levels and XAC(3E) blocked the morphological effects of BDNF, we hypothesized that introduction of 14-3-3 $\zeta$ into growth cones would stabilize pAC and block the effects of BDNF on filopodial dynamics. For these studies, we used the GST-14-3-3 $\zeta$ chimera, which has previously been shown to be functional when expressed in vivo (Gohla and Bokoch, 2002). Incorporation of 14$3-3 \zeta$ alone had no effect on filopodial length but blocked the stimulatory effects of BDNF on filopodial length (Fig. $4 A$ ). Furthermore, 14-3-3 $\zeta$ blocked the BDNF-induced increases in filopodia number (Fig. $4 B$ ). Although 14-3-3 $\zeta$ did not have a significant effect on pAC levels compared with control, 14-3-3 $\zeta$ blocked the BDNF-mediated reduction in pAC levels on retinal growth cones (Fig. 4C). These results further support a role for BDNF signaling through ADF/cofilin, however they do not specifically demonstrate a direct role for $14-3-3 \zeta$ in the BDNF-mediated pathway regulating $\mathrm{pAC}$. 


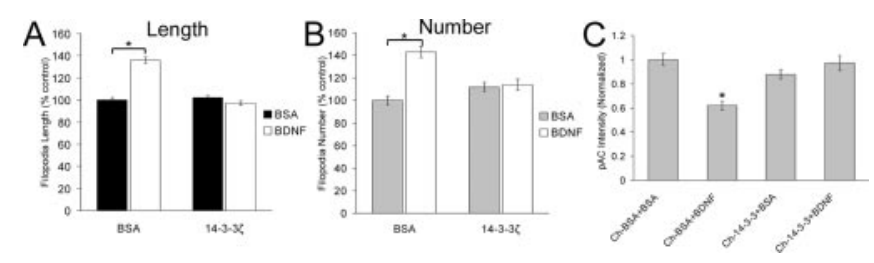

Figure 4. The 14-3-3 $\zeta$ protein blocked the effects of BDNF on filopodial dynamics and pAC levels. The BDNF-induced increases on filopodial length $(A)$ and number $(B)$ were blocked by

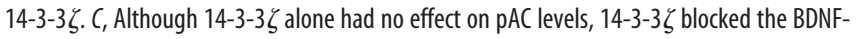
mediated reduction in pAC levels. Data in $A$ and $B$ are percentage of control \pm SEM from six independent experiments. Data in $($ were taken from at least 84 growth cones from four independent experiments. ${ }^{*} p<0.001$; statistical difference relative to control (two-sample $t$ test).

\section{Inhibition of myosin II enhances filopodial length}

If BDNF downregulates RhoA signaling, then a reduction of actomyosin contractility, through lowered ROCK activity, may also contribute to BDNF regulation of filopodial length (Amano et al., 1996; Kimura et al., 1996). Therefore, we tested the effects of inhibition of myosin II on filopodial dynamics. $(S)-(-)$ Blebbistatin has been characterized to specifically inhibit nonmuscle myosin II activity and myosin II-dependent cellular processes (Straight et al., 2003; Kovacs et al., 2004). We found that ( $S)$-( - )-blebbistatin caused a dose-dependent increase in filopodial length on chick retinal growth cones (Fig. 5A-E). Filopodial length on growth cones increased by $\sim 47 \%$ after treatment with $50 \mu \mathrm{M}(S)$-(-)-blebbistatin. A $50 \mu \mathrm{M}$ concentration of blebbistatin caused a modest increase on filopodial number, whereas lower concentrations had no effect on filopodial number (Fig. $5 F$ ). Blebbistatin had similar effects on filopodial length on chick DRG growth cones (our unpublished data).

To determine whether blebbistatin and BDNF have distinct effects on filopodial dynamics, we conducted time-lapse videomicroscopy of retinal growth cone motility. Within 4 min after application of $20 \mu \mathrm{M}(S)-(-)$-blebbistatin, growth cones displayed shrinking of lamellipodia, whereas filopodia detached from the substratum and exhibited bending and buckling behavior (Fig. 5G). Blebbistatin-treated filopodia exhibited enhanced extension rates within 2 min of treatment, as measured by the elongation of filopodial tips, whereas there was a gradual reduction in the rate of retraction (Fig. $5 H$ ). Blebbistatin also caused a reduction in the motility of the growth cone over time. To quantify this observation, we determined a motility index to measure the amount of time filopodia spend moving, as opposed to remaining stationary. Within $15 \mathrm{~min}$, blebbistatin-treated filopodia exhibited an approximate twofold reduction in the motility index relative to before drug treatment (Fig. $5 \mathrm{~K}$ ). In contrast to blebbistatin-treated growth cones, BDNF treatment did not reduce lamellipodial motility, and filopodia did not detach and bend excessively (Fig. 5I). Filopodia treated with BDNF exhibited enhanced extension rates, measured at filopodial tips, but showed no change in the retraction rate (Fig. $5 \mathrm{~J}$ ), as observed previously (Gehler et al., 2004). Also, in contrast to blebbistatin, there was no change in the motility index after treatment with BDNF (Fig. 5K). Thus, our videomicroscopic analysis showed that blebbistatin enhanced filopodial length through increased extension dynamics while reducing the overall motility of filopodia. These morphological effects of blebbistatin on growth cones are distinct from those of BDNF.

\section{BDNF-enhanced filopodial dynamics do not involve regulation of myosin II activity}

We have provided evidence to implicate ADF/cofilin in mediating the effects of BDNF on filopodial dynamics. Whereas inhibition of myosin II with blebbistatin also increased filopodial length, it is not clear whether regulation of myosin II activity is involved in the effects of BDNF. Therefore, we tested the effects of BDNF in combination with blebbistatin on filopodial dynamics. Treatment with BDNF alone for 30 min caused a $29 \%$ increase in filopodial length, whereas treatment with $20 \mu \mathrm{M}$ blebbistatin alone for $15 \mathrm{~min}$ produced a $41 \%$ increase in length (Fig. $6 \mathrm{~A}$ ). Pretreatment with BDNF and then the addition of blebbistatin produced a $56 \%$ increase on filopodial length. These additive effects of BDNF and blebbistatin suggest BDNF may mediate its effects through a mechanism that is independent of myosin II activity.

Analysis of filopodial number provided additional evidence that the effects of BDNF are myosin II independent. BDNF alone caused a $40 \%$ increase in filopodial number (Fig. 6B). Although $20 \mu \mathrm{M}$ blebbistatin alone had no effect on filopodial number, simultaneous exposure to BDNF and blebbistatin resulted in a similarly increased number of filopodia as BDNF treatment alone. These results suggest that reduced myosin II activity is not involved in the BDNF-induced increases on filopodial number.

To test further whether BDNF signals through a myosin IIindependent pathway, we determined the effects of the ROCK inhibitor Y-27632 and blebbistatin on filopodial dynamics. BDNF regulation of filopodia is mimicked by inhibition of ROCK (Fig. 1), and, furthermore, inhibiting ROCK downregulates $\mathrm{pAC}$ in a manner similar to BDNF treatment (Fig. 3J-P). It has been suggested that Y-27632 inhibits myosin IIa-mediated neurite retraction in neuroblastoma cells (Chantler and Wylie, 2003). To determine whether Y-27632 mediates its effects on filopodial dynamics independently of myosin II inhibition, chick retinal cultures were simultaneously treated with $10 \mu \mathrm{M}$ Y-27632 and $20 \mu \mathrm{M}$ blebbistatin. Treatment with $10 \mu \mathrm{M}$ Y-27632 alone caused an $\sim 31 \%$ and $\sim 25 \%$ increase on filopodial length and number, respectively, whereas growth cones treated with $20 \mu \mathrm{M}$ blebbistatin alone displayed an $\sim 49 \%$ and $\sim 12 \%$ enhancement on filopodial length and number (Fig. 6C,D). Simultaneous treatment with Y-27632 and blebbistatin had additive effects by causing an $\sim 86 \%$ increase on filopodial length, whereas filopodial number was similar to Y-27632 treatment alone. These results suggest that Y-27632 does not enhance filopodial length through inhibition of myosin II and provide additional evidence that BDNF signals through a myosin II-independent pathway.

Finally, we tested the effects of myosin II inhibition on growth cones loaded with ADF/cofilin mutant proteins. This experimental approach tested whether blebbistatin treatment requires $\mathrm{ADF} /$ cofilin activity to mediate its effects, and because XAC(3E) completely blocked the BDNF-mediated enhancement on filopodial dynamics, it will help further characterize whether the effects of BDNF involve a reduction in myosin II activity. Whereas the effects of BDNF were blocked by $\mathrm{XAC}(3 \mathrm{E})$, treatment of $\mathrm{XAC}(3 \mathrm{E})$-loaded growth cones with $20 \mu \mathrm{M}$ blebbistatin increased filopodial length and number by $\sim 48 \%$ and $19 \%$, respectively, similar to blebbistatin treatment alone (Fig. 6E,F). Similarly, although the enhancement of filopodial length and number in $\mathrm{XAC}(3 \mathrm{~A})$-treated growth cones was not further increased by BDNF, the addition of blebbistatin to XAC(3A)-loaded cultures did produce additive effects on both filopodial length and number. These studies combining ADF/cofilin mutants with blebbistatin show that the effects of blebbistatin are independent of 
ADF/cofilin activity. Furthermore, because the effects of BDNF are blocked by $\mathrm{XAC}(3 \mathrm{E})$, whereas the effects of blebbistatin are not influenced by $\mathrm{XAC}(3 \mathrm{E})$, these results further indicate that BDNF does not signal through myosin II inhibition.

\section{Discussion}

Filopodia are dynamic cellular extensions with key roles in morphogenetic events (Wood and Martin, 2002). The critical sensory role of filopodia in neuronal growth cones is indicated by studies showing that filopodial inhibition disrupts pathfinding in vivo and in vitro (Bentley and Toroian-Raymond, 1986; Chien et al., 1993; Zheng et al., 1996). Furthermore, growth cone turning toward a chemoattractant is initiated by an asymmetrical distribution of filopodia (Zheng et al., 1996). Filopodial signaling may initiate growth cone responses to extracellular ligands (Robles et al., 2003; Yuan et al., 2003). In addition, interactions between axonal and dendritic filopodia are important during morphogenesis of axonal and dendritic arbors and in synaptogenesis (for review, see Jontes and Smith, 2000; Niell et al., 2004).

BDNF is implicated in regulating axonal and dendritic morphogenesis (McAllister et al., 1999; Alsina et al., 2001; Chao, 2003). We previously showed that neurotrophin stimulation of filopodial number and length is mediated by p75-dependent downregulation of RhoA activity in retinal growth cones (Gehler et al., 2004). A 30\% increase in filopodial length expands the search volume of a growth cone by $120 \%$. Such enhanced sensory capability may facilitate detection of morphogenetic gradients (Isbister et al., 2003). In addition, filopodia are important for the ability of growth cones to cross nonadhesive regions separating adhesive substrata (Hammarback and Letourneau, 1986; Clark et al., 1993). Consequently, longer filopodia may increase the abilities of growth cones to detect guidance or morphogenetic information on cells and extracellular surfaces.

We have shown that inhibition of the RhoA effector ROCK mimics the effects of BDNF on retinal growth cone filopodial dynamics. Second, we have shown that $\mathrm{ADF} /$ cofilin activity is sufficient and necessary to transduce the effects of BDNF on filopodial dynamics. Third, BDNF promotes the dephosphorylation, and therefore activation, of ADF/cofilin. Finally, inhibition of myosin II enhances filopodial length, but myosin II activity is not involved in mediating the effects of BDNF on filopodial length or number.

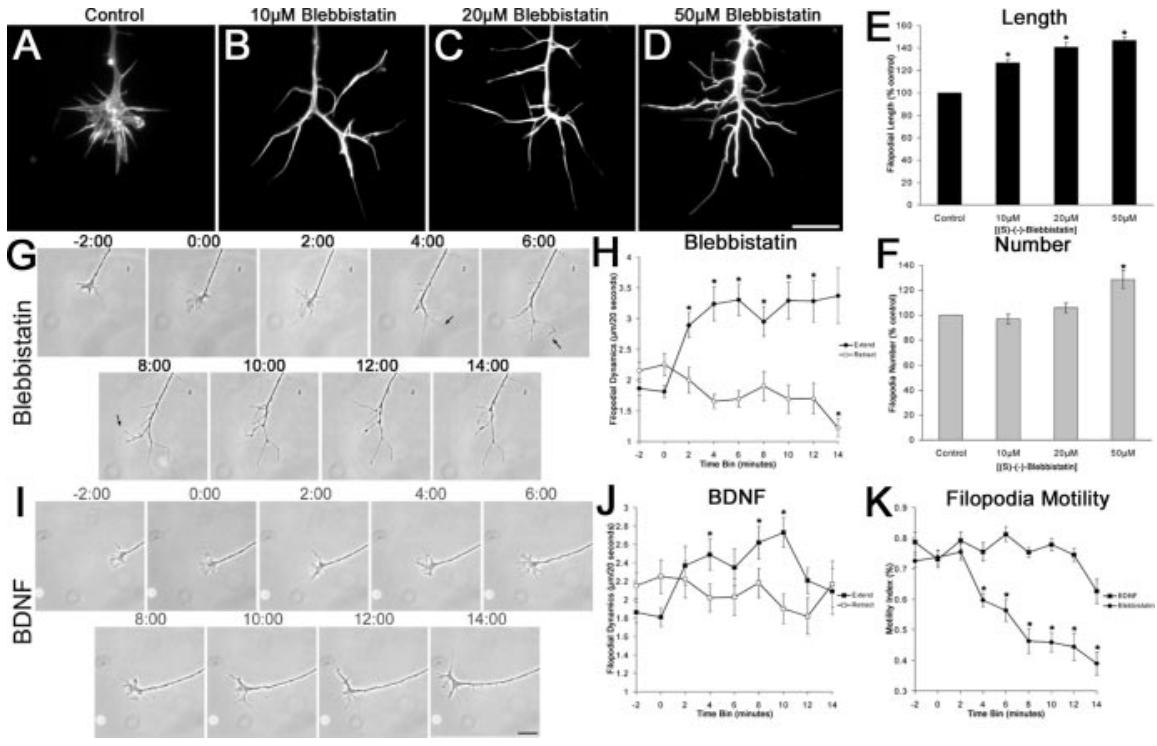

Figure 5. Inhibition of myosin II enhanced filopodial length, but growth cones exhibit morphological differences compared with BDNF treatment. $A-D,(S)-(-)$-Blebbistatin had a dose-dependent effect on filopodial length and number of retinal growth cones stained using Alexa568-conjugated phalloidin. $E$, A $50 \mu \mathrm{m}$ concentration of blebbistatin increased filopodial length by $\sim 47 \%$. F, A $50 \mu$ m concentration of blebbistatin had a small effect on filopodial number, whereas lower concentrations had no effect. $G$, Time-lapse images of a growth cone treated with $20 \mu \mathrm{m}$ blebbistatin. The growth cone displayed normal motility before the addition of blebbistatin (-2:00). After the addition of blebbistatin (0:00), the lamellipodia retracted, whereas filopodia increased in length (4:00). There was a tendency for filopodia to detach and buckle after treatment with blebbistatin (arrows). $H$, Blebbistatin increased filopodia extension rates while causing a gradual reduction in the retraction rates. $I$, Time-lapse images of a BDNF-treated growth cone. Four minutes after the addition of $160 \mathrm{~nm}$ BDNF (4:00), filopodia length and number increased, whereas the lamellipodia remained intact. J, BDNF increased extension rates while having no effect on the retraction rates. $K$, Filopodia treated with blebbistatin exhibited a twofold reduction in the motility index, whereas BDNF-treated filopodia exhibited no change in filopodial motility. Filopodia extension and retraction dynamics were pooled into $2 \mathrm{~min}$ time bins. Scale bars, $10 \mu \mathrm{m}$. Data are percentage of control \pm SEM from four independent experiments. Videomicroscopy results were obtained from at least six growth cones for each treatment. ${ }^{*} p<0.001$; statistical significance relative to control (two-sample $t$ test).
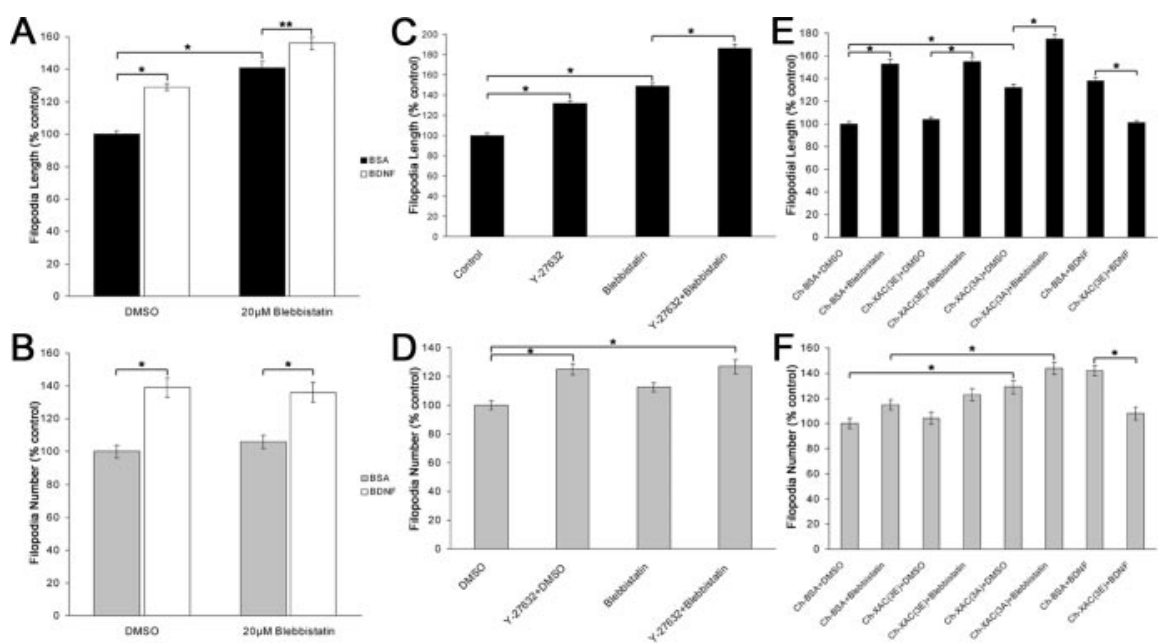

Figure 6. BDNF does not signal through myosin II to regulate filopodial dynamics. BDNF has additive effects on filopodial length $(A)$ and number $(B)$ on blebbistatin-treated growth cones. C, A $10 \mu \mathrm{m}$ concentration of Y-27632 or $20 \mu \mathrm{m}$ blebbistatin caused an $\sim 31$ and $\sim 49 \%$ increase on filopodial length, respectively, whereas treatment with both Y-27632 and blebbistatin produced an $86 \%$ increase on filopodial length. D, Y-27632 and blebbistatin enhanced filopodial number by $\sim 25$ and $12 \%$, respectively. Treatment with both Y-27632 and blebbistatin enhanced filopodial number to similar levels as Y-27632 treatment alone. $E$, The effects of blebbistatin do not require ADF/cofilin activity. Contrary to BDNF treatment, XAC( $3 E)$ had no effect on blebbistatin-induced increases on length. In addition, treatment of $X A C(3 A)$ cultures with blebbistatin exhibited additive effects on length. $F$, Blebbistatin treatment of $X A C(3 E)$-loaded growth cones caused a similar increase in filopodial number as blebbistatin treatment alone, whereas blebbistatin had additive effects on the number of filopodia on XAC(3A)-treated growth cones. Data are percentage of control \pm SEM from a minimum of four independent experiments. ${ }^{*} p<0.001$; ${ }^{* *} p<0.005$; statistical difference (two-sample $t$ test). 
Our results with three ROCK inhibitors indicate that reduced ROCK signaling stimulates filopodial formation, similar to the effect of ROCK inhibition on neurite formation (Bito et al., 2000). ROCK inhibitors replicate the stimulation of filopodial length by BDNF and are not additive with BDNF. However, ROCK inhibitors have a lesser effect on filopodial number, which is increased by cotreatment with BDNF to the level of BDNF treatment alone. BDNF may regulate filopodial number through additional pathways.

$\mathrm{ADF} /$ cofilin plays a key role in promoting actin filament dynamics by severing F-actin to generate barbed ends for filament elongation and by enhancing the release of G-actin from F-actin pointed ends. Expression of activated cofilin increased barbed filament ends and F-actin content to promote motility of MTLn3 cells (Ghosh et al., 2004). ADF/cofilin is regulated by RhoA signaling through the ROCK effector LIMK (Sarmiere and Bamburg, 2004), which inhibits $\mathrm{ADF} /$ cofilin via phosphorylation at serine 3 (Arber et al., 1998; Yang et al., 1998; Meng et al., 2002). LIMK overexpression depresses growth cone motility and neurite extension in sensory neurons (Aizawa et al., 2001; Endo et al., 2003). The inhibitory effects of LIMK are opposed by the ADF/ cofilin phosphatase slingshot (SHH) (Endo et al., 2003; NagataOhashi et al., 2004; Sarmiere and Bamburg, 2004). Supplemental material (available at www.jneurosci.org as supplemental material) demonstrates the presence of SSH in retinal growth cones.

With a peptide-based delivery system, we introduced exogenous proteins into growth cones. Incorporation of constitutively active $\mathrm{ADF} /$ cofilin [XAC(3A)] increased filopodial length to similar levels as BDNF treatment, indicating that increased ADF/ cofilin activity is sufficient to increase filopodial length. $\mathrm{XAC}(3 \mathrm{~A})$ had modest effects on filopodial number. The addition of BDNF to $\mathrm{XAC}(3 \mathrm{~A})$-treated neurons did not further increase filopodia length but increased filopodial number the same as BDNF treatment alone. Conversely, pseudo-phosphorylated (inactive) ADF/ cofilin, XAC(3E), blocked the BDNF-induced increase in length and number, indicating $\mathrm{ADF} /$ cofilin activity is required for BDNF to stimulate filopodial dynamics. A nonsevering mutant of $\mathrm{ADF} /$ cofilin also blocked the effects of BDNF. Consistent with a requirement for active $\mathrm{ADF} /$ cofilin to mediate the effects of BDNF, BDNF caused a reduction in the amount of inactive phospho-ADF/cofilin in growth cones. This was mimicked by inhibition of ROCK and is consistent with the finding that Y-27632 reduced pAC levels in THP-1 monocytes (Worthylake and Burridge, 2003). Incorporation of $14-3-3 \zeta$, which binds and stabilizes inactive phospho-ADF/cofilin, blocked BDNF-induced increases on filopodial length and number. The $14-3-3 \zeta$ protein produced no change in pAC levels compared with control but did suppress the BDNF-induced reduction in pAC staining. Collectively, these results show that BDNF regulates filopodial dynamics by increasing the activity of ADF/cofilin.

How do pseudo-phosphorylated $\mathrm{ADF} /$ cofilin $[\mathrm{XAC}(3 \mathrm{E})]$ or nonsevering $\mathrm{ADF} /$ cofilin block the effects of BDNF in growth cones that contain endogenous ADF/cofilin? Perhaps XAC(3E) and nonsevering $\mathrm{ADF} / \mathrm{cofilin}$ bind $\mathrm{SHH}$ or other phosphatases and interfere with dephosphorylation to activate endogenous $\mathrm{ADF} /$ cofilin. Expressed XAC(3E) also acted in a dominantnegative manner on fibroblast polarity (Dawe et al., 2003). That a nonsevering mutant of ADF/cofilin blocked BDNF-induced increases of filopodial length and number indicates that actin filament severing by ADF/cofilin to generate new barbed filament ends is important for promoting actin dynamics (Endo et al., 2003). Additional information on the mechanism by which ADF/ cofilin regulates filopodial dynamics will come from tracking ac- tin dynamics in filopodia using speckle microscopy or photobleaching (Watanabe and Mitchison, 2002).

ROCK also activates myosin II (Amano et al., 1996; Kimura et al., 1996), raising the possibility that the effects of BDNF on filopodia involves myosin II. Lin et al. (1996) showed that filopodial elongation is negatively regulated by myosin-driven retrograde F-actin flow. However, Diefenbach et al. (2002) found that chromophore-assisted laser inactivation (CALI) of myosin II did not affect filopodial dynamics and growth cones from myosin IIB knock-out mice showed normal filopodial dynamics (Brown and Bridgman, 2003). Furthermore, neurons express two main isoforms of myosin II that may regulate distinct aspects of neuronal morphology (Wylie and Chantler, 2003).

We found that, like BDNF treatment, treatment with the myosin II-specific inhibitor blebbistatin (Kovacs et al., 2004) increased filopodial length and $50 \mu \mathrm{m}$ of blebbistatin modestly increased filopodial number. Blebbistatin had distinct effects on filopodial motility compared with BDNF. Both blebbistatin and BDNF augmented filopodial extension rates, but blebbistatin significantly reduced filopodial motility, whereas BDNF produced no change in motility. In addition, filopodia of growth cones treated with blebbistatin detached from the substratum and exhibited bending and buckling, whereas lamellipodia retracted. These observations resemble the finding that disruption of myosin IIA reduced cell adhesion of mouse neuroblastoma cells (Wylie and Chantler, 2001). Furthermore, CALI of vinculin, a component of focal contacts, in growth cones also caused filopodia to detach and buckle (Sydor et al., 1996). Thus, myosin II inhibition with blebbistatin may disrupt adhesion of filopodia, and we speculate the increased filopodial extension rates are attributable to reduced membrane cortical tension and altered actin dynamics (Mallavarapu and Mitchison, 1999; Raucher and Sheetz, 2000).

Our results indicated myosin II is not involved in mediating the effects of BDNF on filopodial dynamics. Inhibition of myosin II with blebbistatin was additive with either BDNF or inhibition of ROCK in stimulating filopodial length. In contrast, blocking $\mathrm{ADF} /$ cofilin activity with $\mathrm{XAC}(3 \mathrm{E})$, which inhibited BDNFinduced increase of filopodial length, does not inhibit the enhancement of filopodial length by blebbistatin. Furthermore, the stimulation of filopodial length by constitutively active $\mathrm{XAC}(3 \mathrm{~A})$, which is not additive with BDNF treatment, is additive with blebbistatin treatment. Together these results indicate that BDNF regulates filopodial dynamics independently of myosin II. Although we cannot rule out the contributions to filopodial dynamics of other myosins, particularly myosin IC, which may be involved in retrograde flow and lamellipodial spread (Diefenbah et al., 2002), and myosin V (Wang et al., 1996), only myosin II is known to be regulated by ROCK. Furthermore, myosin V null mice display normal growth cone morphology (Evans et al., 1997).

BDNF regulates many aspects of the behavior of retinal growth cones and axonal terminals. Multiple signaling pathways and multiple receptors are involved (Chao, 2003; Song and Poo, 2001), including mechanisms that require protein synthesis and minimum time intervals (Gallo et al., 2002a). These diverse activities are complex, involving many proteins and interactions. We limited this study to investigating the short-term effects of BDNF on filopodial dynamics. Our results clearly demonstrate that $\mathrm{ADF} /$ cofilin mediates the effects of BDNF on filopodial dynamics, whereas regulation of myosin II activity is not involved. It is becoming apparent that $\mathrm{ADF} /$ cofilin is a focal point for regulating growth cone actin dynamics through diverse mechanisms. 
LIMK, SHH, and 14-3-3 $\zeta$ all have roles in regulating the balance of active versus inactive ADF/cofilin, and all have been demonstrated to modulate growth cone behaviors (Aizawa et al., 2001; Endo et al., 2003; Niwa et al., 2002). Furthermore, 14-3-3 $\zeta$ binds not only to phospho-ADF/cofilin (Gohla and Bokoch, 2002) but also to LIMK (Birkenfeld et al., 2003) and SHH (Nagata-Ohashi et al., 2004), providing additional complexity in regulating ADF/ cofilin activity. In conclusion, we present evidence that ADF/ cofilin has an important role in mediating the effects of neurotrophins, an important class of axonal guidance and morphogenetic cues, on filopodial dynamics of retinal neuronal growth cones. Together with evidence that ADF/cofilin is involved in mediating the effects of other guidance cues (Aizawa et al., 2001), our results emphasize the significance of ADF/cofilin as a focus of signaling to regulate growth cone pathfinding and morphogenesis.

\section{References}

Abe H, Obinata T, Minamide LS, Bamburg JR (1996) Xenopus laevis actindepolymerizing factor/cofilin: a phosphorylation-regulated protein essential for development. J Cell Biol 132:871-885.

Agnew BJ, Minamide LS, Bamburg JR (1995) Reactivation of phosphorylated actin depolymerizing factor and identification of the regulatory site. J Biol Chem 270:17582-17587.

Aizawa H, Wakatsuki S, Ishii A, Moriyama K, Sasaki Y, Ohashi K, SekineAizawa Y, Sehara-Fujisawa A, Mizuno K, Goshima Y, Yahara I (2001) Phosphorylation of cofilin by LIM-kinase is necessary for semaphorin 3A-induced growth cone collapse. Nat Neurosci 4:367-373.

Alsina B, Vu T, Cohen-Cory S (2001) Visualizing synapse formation in arborizing optic axons in vivo: dynamics and modulation by BDNF. Nat Neurosci 4:1093-1101.

Amano M, Ito M, Kimura K, Fukata Y, Chihara K, Nakano T, Matsuura Y, Kaibuchi K (1996) Phosphorylation and activation of myosin by Rhoassociated kinase (Rho-kinase). J Biol Chem 271:20246-20249.

Arber S, Barbayannis FA, Hanser H, Schneider C, Stanyon CA, Bernard O, Caroni P (1998) Regulation of actin dynamics through phosphorylation of cofilin by LIM-kinase. Nature 393:805-809.

Bamburg JR (1999) Proteins of the ADF/cofilin family: essential regulators of actin dynamics. Annu Rev Cell Dev Biol 15:185-230.

Bentley D, Toroian-Raymond A (1986) Disoriented pathfinding by pioneer neurone growth cones deprived of filopodia by cytochalasin treatment. Nature 323:712-715.

Birkenfeld J, Betz H, Roth D (2003) Identification of cofilin and LIMdomain-containing protein kinase 1 as novel interaction partners of 143-3. Biochem J 369:45-54.

Bito H, Furuyashiki T, Ishihara H, Shibasaki Y, Ohashi K, Mizuno K, Maekawa M, Ishizaki T, Narumiya S (2000) A critical role for a Rhoassociated kinase, p160ROCK, in determining axon outgrowth in mammalian CNS neurons. Neuron 26:431-441.

Bobkov AA, Muhlrad A, Shvetsov A, Benchaar S, Scoville D, Almo SC, Reisler E (2004) Cofilin (ADF) affects lateral contacts in F-actin. J Mol Biol 337:93-104.

Bridgman PC, Dave S, Asnes CF, Tullio AN, Adelstein RS (2001) Myosin IIB is required for growth cone motility. J Neurosci 21:6159-6169.

Brown MD, Bridgman PC (2003) Retrograde flow rate is increased in growth cones from myosin IIB knockout mice. J Cell Sci 116:1087-1094.

Carlier M-F, Laurent V, Santolini J, Melki R, Didry D, Xia G-X, Hong Y, Chua $\mathrm{N}-\mathrm{H}$, Pantaloni D (1997) Actin depolymerizing factor (ADF/cofilin) enhances the rate of filament turnover: implication in actin-based motility. J Cell Biol 136:1307-1323.

Chantler PD, Wylie SR (2003) Elucidation of the separate roles of myosins IIA and IIB during neurite outgrowth, adhesion and retraction. IEE Proc Nanobiotechnol 150:111-125.

Chao MV (2003) Neurotrophins and their receptors: a convergence point for many signalling pathways. Nat Rev Neurosci 4:299-309.

Chien C-B, Rosenthal DE, Harris WA, Holt CE (1993) Navigational errors made by growth cones without filopodia in the embryonic Xenopus brain. Neuron 11:237-251.

Clark P, Britland S, Connolly P (1993) Growth cone guidance and neuron morphology on micropatterned laminin surfaces. J Cell Sci 105:203-212.
Davenport RW, Dou P, Rehder V, Kater SB (1993) A sensory role for neuronal growth cone filopodia. Nature 361:721-724.

Dawe HR, Minamide LS, Bamburg JR, Cramer LP (2003) ADF/cofilin controls cell polarity during fibroblast migration. Curr Biol 13:252-257.

Dickson BJ (2001) Rho GTPases in growth cone guidance. Curr Opin Neurobiol 11:103-110.

Dickson BJ (2002) Molecular mechanisms of axon guidance. Science 298:1959-1964.

Diefenbach TJ, Latham VM, Yimlamai D, Liu CA, Herman IM, Jay DG (2002) Myosin 1c and myosin IIB serve opposing roles in lamellipodial dynamics of the neuronal growth cone. J Cell Biol 158:1207-1217.

Endo M, Ohashi K, Sasaki Y, Goshima Y, Niwa R, Uemura T, Mizuno K (2003) Control of growth cone motility and morphology by LIM kinase and Slingshot via phosphorylation and dephosphorylation of cofilin. J Neurosci 23:2527-2537.

Ernst AF, Gallo G, Letourneau PC, McLoon SC (2000) Stabilization of growing retinal axons by the combined signaling of nitric oxide and brain-derived neurotrophic factor. J Neurosci 20:1458-1469.

Evans LL, Hammer J, Bridgman PC (1997) Subcellular localization of myosin $\mathrm{V}$ in nerve growth cones and outgrowth from dilute-lethal neurons. J Cell Sci 110:439-449.

Fu H, Subramanian RR, Masters SC (2000) 14-3-3 proteins: structure, function, and regulation. Annu Rev Pharmacol Toxicol 40:617-647.

Galkin VE, Orlova A, Lukoyanova N, Wriggers W, Egelman EH (2001) Actin depolymerizing factor stabilizes an existing state of F-actin and can change the tilt of F-actin subunits. J Cell Biol 153:75-86.

Gallo G, Lefcort FB, Letourneau PC (1997) The trkA receptor mediates growth cone turning toward a localized source of nerve growth factor. J Neurosci 17:5445-5454.

Gallo G, Ernst AF, McLoon SC, Letourneau PC (2002a) Transient PKA activity is required for initiation but not for maintenance of BDNFmediated protection from nitric oxide induced growth cone collapse. J Neurosci 22:5016-5023.

Gallo G, Yee Jr HF, Letourneau PC (2002b) Actin turnover is required to prevent axon retraction driven by endogenous actomyosin contractility. J Cell Biol 158:1219-1228.

Gehler S, Gallo G, Veien E, Letourneau PC (2004) p $75^{\text {NTR }}$ signaling regulates growth cone filopodial dynamics through modulating RhoA activity. J Neurosci 24:4363-4372.

Ghosh M, Song X, Mouneimne G, Sidani M, Lawrence DS, Condeelis JS (2004) Cofilin promotes actin polymerization and defines the direction of cell motility. Science 304:743-746.

Glebova NO, Ginty DD (2004) Heterogeneous requirement of NGF for sympathetic target innervation in vivo. J Neurosci 24:743-751.

Gohla A, Bokoch GM (2002) 14-3-3 regulates actin dynamics by stabilizing phosphorylated cofilin. Curr Biol 12:1704-1710.

Gomez TM, Letourneau PC (1994) Filopodia initiate choices made by sensory neuron growth cones at laminin/fibronectin borders in vitro. J Neurosci 14:5959-5972.

Gundersen RW, Barrett JN (1979) Neuronal chemotaxis: chick dorsal-root axons turn toward high concentrations of nerve growth factor. Science 206:1079-1080.

Hammarback JA, Letourneau PC (1986) Neurite extension across regions of low cell-substratum adhesion. Implications for the guidepost theory of axonal pathfinding. Dev Biol 117:655-662.

Isbister CM, Mackenzie PJ, To KC, O’Connor TP (2003) Gradient steepness influences pathfinding decisions of neuronal growth cones in vivo. J Neurosci 23:193-202.

Jontes JD, Smith SJ (2000) Filopodia, spines, and the generation of synaptic diversity. Neuron 27:11-14.

Kimura K, Ito M, Amano M, Chihara K, Fukata Y, Nakafuku M, Yamamori B, Feng J, Nakano T, Okawa K, Iwamatsu A, Kaibuchi K (1996) Regulation of myosin phosphatase by Rho and Rho-associated kinase (Rho-kinase). Science 273:245-248.

Kovacs M, Toth J, Hetenyi C, Malnasi-Csizmadia A, Sellers JR (2004) Mechanism of belbbistatin inhibition of myosin II. J Biol Chem 279:35557-35563.

Kuhn TB, Schmidt MF, Kater SB (1994) Laminin and fibronectin guideposts signal sustained but opposite effects to passing growth cones. Neuron 14:275-285.

Lin CH, Espreafico EM, Mooseker MS, Forscher P (1996) Myosin drives retrograde F-actin flow in neuronal growth cones. Neuron 16:769-782. 
Maciver SK (1998) How ADF/cofilin depolymerizes actin filaments. Curr Biol 10:140-144.

Maekawa M, Ishizaki T, Boku S, Watanabe N, Fujita A, Iwamatsu A, Obinata T, Ohashi K, Mizuno K, Narumiya S (1999) Signaling from Rho to the actin cytoskeleton through protein kinases ROCK and LIM-kinase. Science 285:895-898.

Mallavarapu A, Mitchison T (1999) Regulated actin cytoskeleton assembly at filopodium tips controls their extension and retraction. J Cell Biol 146:1097-1106.

McAllister AK, Katz LC, Lo DC (1999) Neurotrophins and synaptic plasticity. Annu Rev Neurosci 22:295-318.

McGough A, Pope B, Chiu W, Weeds A (1997) Cofilin changes the twist of F-actin: implications for actin filament dynamics and cellular function. J Cell Biol 138:771-781.

McQuillen PS, DeFreitas MF, Zada G, Shatz CJ (2002) A novel role for p $75^{\text {NTR }}$ in subplate growth complexity and visual thalamocortical innervation. J Neurosci 22:3580-3593.

Meberg PJ, Bamburg JR (2000) Increase in neurite outgrowth mediated by overexpression of actin depolymerizing factor. J Neurosci 20:2459-2469.

Meberg PJ, Ono S, Minamide LS, Takahashi M, Bamburg JR (1998) Actin depolymerizing factor and cofilin phophorylation dynamics: response to signals that regulate neurite extension. Cell Motil Cytoskeleton 39:172-190.

Meng Y, Zhang Y, Tregoubov V, Janus C, Cruz L, Jackson M, Lu W-Y, MacDonald JF, Wang JY, Falls DL, Jia Z (2002) Abnormal spine morphology and enhanced LTP in LIMK-1 knockout mice. Neuron 35:121-144.

Nagata-Ohashi K, Ohta Y, Goto K, Mori R, Nishita M, Ohashi K, Kousaka K, Iwamatsu A, Niwa R, Uemura T, Mizuno K (2004) A pathway of neuregulin-induced activation of cofilin-phosphatase Slingshot and cofilin in lamellipodia. J Cell Biol 165:465-471.

Niell CM, Meyer MP, Smith SJ (2004) In vivo imaging of synapse formation on a growing dendritic arbor. Nat Neurosci 7:254-260.

Niwa R, Nagata-Ohashi K, Takeichi M, Mizuno K, Uemura T (2002) Control of actin reorganization by Slingshot, a family of phosphatases that dephosphorylate ADF/cofilin. Cell 108:233-246.

O'Connor TP, Duerr JS, Bentley D (1990) Pioneer growth cone steering decisions mediated by single filopodial contacts in situ. J Neurosci 10:3935-3948.

Pope BJ, Gonsior SM, Yeoh S, McGough A, Weeds AG (2000) Uncoupling actin filament fragmentation by cofilin from increased subunit turnover. J Mol Biol 298:649-661.
Raucher D, Sheetz MP (2000) Cell spreading and lamellipodial extension rate is regulated by membrane tension. J Cell Biol 148:127-136.

Robles E, Huttenlocher A, Gomez TM (2003) Filopodial calcium transients regulate growth cone motility and guidance through local activation of calpain. Neuron 38:597-609.

Sarmiere PD, Bamburg JR (2004) Regulation of the neuronal actin cytoskeleton by ADF/cofilin. J Neurobiol 58:103-117.

Song H-J, Poo M-m (2001) The cell biology of neuronal navigation. Nat Cell Biol 3:E81-E88.

Straight AF, Cheung A, Limouze J, Chen I, Westwood NJ, Sellers JR, Mitchison TJ (2003) Dissecting temporal and spatial control of cytokinesis with a myosin II inhibitor. Science 299:1743-1747.

Sydor AM, Su AL, Wang F-S, Xu A, Jay DG (1996) Talin and vinculin play distinct roles in filopodial motility in the neuronal growth cone. J Cell Biol 134:1197-1207.

Tucker KL, Meyer M, Barde Y-A (2001) Neurotrophins are required for nerve growth during development. Nat Neurosci 4:29-37.

Wang F-S, Wolenski JS, Cheney RE, Mooseker MS, Jay DG (1996) Function of myosin- $\mathrm{V}$ in filopodial extension of neuronal growth cones. Science 273:660-663.

Watanabe N, Mitchison TJ (2002) Single-molecule speckle analysis of actin filament turnover in lamellipodia. Science 295:1083-1086.

Wood W, Martin P (2002) Structures in focus-filopodia. Int J Biochem Cell Biol 34:726-730.

Worthylake RA, Burridge K (2003) RhoA and ROCK promote migration by limiting membrane protrusions. J Biol Chem 278:13578-13584.

Wylie SR, Chantler PD (2001) Separate but linked functions of conventional myosins modulate adhesion and neurite outgrowth. Nat Cell Biol 3:88-92.

Wylie SR, Chantler PD (2003) Myosin IIA drives neurite retraction. Mol Biol Cell 14:4654-4666.

Yang N, Higuchi O, Ohashi K, Nagata K, Wada A, Kangawa K, Nishida E, Mizuno K (1998) Cofilin phosphorylation by LIM-kinase 1 and its role in Rac-mediated actin reorganization. Nature 393:809-812.

Yuan X-b, Jin M, Xu X, Song Y-q, Wu C-p, Poo M-m, Duan S (2003) Signaling and crosstalk of Rho GTPases in mediating axon guidance. Nat Cell Biol 5:1-8.

Zheng JQ, Wan J-j, Poo M-m (1996) Essential role of filopodia in chemotropic turning of nerve growth cone induced by a glutamate gradient. J Neurosci 16:1140-1149. 